\title{
EL RECHAZO DE CARL MENGER A LA ECONOMÍA MATEMÁTICA. UNA APROXIMACIÓN
}

\author{
MARÍA BLANCO GONZÁLEZ*
}

Resumen: El objetivo de este documento de trabajo es, por un lado, analizar los motivos que llevaron al fundador de la Escuela Austriaca, Carl Menger, a rechazar el empleo de las matemáticas como herramienta de la teoría económica y el alcance de esta oposición. Por otra parte, se trata de considerar si actualmente esta postura metodológica sigue siendo válida o, por el contrario, los avances en la matemática permiten un empleo más amplio en la teoría económica manteniendo los postulados mengerianos.

Hoy en día, la matemática también ha evolucionado y han aparecido disciplinas como la Teoría de la Decisión Multicriterio que intenta solucionar los problemas que afloran en la toma de decisiones cuando existen objetivos múltiples y, a menudo, conflictivos entre sí. Estos avances ponen sobre la mesa el recalcitrante rechazo hacia las matemáticas, no tanto de Menger, como de sus seguidores.

Palabras clave: Metodología de la economía, Escuela Austriaca de Economía, Carl Menger, economía matemática, Teoría de la Decisión Multicriterio.

Abstract: This article tries, on the one hand, to analyse why Carl Menger, the Austrian School of Economics founder, rejected the use of mathematics as a tool for economic theory and the scope of his opposition. On the other hand, the article considers the advances in mathematical science in order to question at what point Menger's methodology is still sounding, or on the contrary, if these new developments allow a wider use of mathematical economics maintaining, at the same time, the Austrian methodological assumptions.

Nowadays, the Multicriteria Decision Theory has emerged as a powerful tool to assist in the process of searching for decisions, which best satisfy a multitude of conflicting objectives. These advancements may question the

* Universidad CEU-San Pablo. Insituto Juan de Mariana. Correo: mariabg@ceu.es 
persistent opposition to mathematical economics not so much from Carl Menger but from his successors.

Key words: Economic methodology, Austrian School of Economics, Carl Menger, mathematical economics, Multicriteria Decision Theory.

JEL Classification: B1, B2, B4, C6, C8.

I.

\section{INTRODUCTION}

El objetivo de este artículo es, por un lado, analizar los motivos que llevaron al fundador de la Escuela Austriaca, Carl Menger, a rechazar el empleo de las matemáticas como herramienta de la teoría económica y el alcance de esta oposición. Por otra parte, se trata de considerar si actualmente esta postura metodológica sigue siendo válida o, por el contrario, los avances en la matemática permiten un empleo más amplio en la teoría económica manteniendo los postulados mengerianos.

En la sección I, se analizan las similitudes y diferencias principales entre Menger y los otros dos marginalistas, Walras y Jevons. La sección II se centra en un estudio pormenorizado de la metodología de Menger haciendo referencia especialmente a su opinión respecto al empleo de las matemáticas en la investigación económica. La tercera sección expone la formación matemática de este autor. La sección IV consiste en una análisis de algunas aportaciones matemáticas a la economía más recientes y se cuestiona si se adecuan o no a la metodología de los primeros austriacos. La V sección analiza la herencia metodológica que sus dos principales discípulos recibieron de Menger. Para terminar se exponen las conclusiones.

II.

\section{CARL MENGER Y LA REVOLUCIÓN MARGINAL}

La Escuela Austriaca de pensamiento económico que surgió en Viena a partir del último cuarto del siglo XIX, se caracteriza por su preocupación por el método y el campo de estudio de la teoría 
económica y tradicionalmente se la considera uno de los principales oponentes a la matematización de la economía. Aunque estos autores tenían una concepción apriorística y deductiva de la teoría pura de la economía, no contemplaban el uso de las matemáticas más que de forma auxiliar. En contra de lo que suele pensarse, no las rechazaban totalmente sino que consideraban el ámbito de su aplicación muy reducido, y por tanto les restaban importancia. Sin embargo, esta opinión varía con el paso del tiempo.

Ésta Escuela nació alrededor de la figura de Carl Menger, uno de los tres pioneros de la llamada Revolución Marginal, junto con el francés Léon Walras y el británico William Stanley Jevons. Se denomina Revolución Marginal al surgimiento de una teoría del valor basada en la Ley de Utilidad Marginal Decreciente de forma simultánea e independiente por los tres autores mencionados, contrapuesta a la teoría del valor clásica basada en el coste de producción, y que tuvo lugar en la década de 1870. Esta teoría derivaba en una concepción de la producción como un tipo de intercambio y rompía con la tradición de los economistas clásicos. Este nuevo enfoque de la economía es el germen de la corriente neoclásica.

Habitualmente se asocian los nombres de Menger, Jevons y Walras sin especificar las diferencias que hay entre sus teorías. Incluso se consideran las teorías de la Escuela Austriaca como una versión no matemática de las de Walras y Jevons (Bousquet, G.-H., 1924, pp. 840 y ss.; Bloch, H.-S., 1940 reimpreso en Blaug, 1990, p. 429; Kirzner, I.M., 1987, p. 146; Seligman, B.B., 1963, p. 271).

Erich Streissler, historiador del pensamiento económico austriaco, considera que el objetivo de la teoría económica para los marginalistas neoclásicos era la investigación de las condiciones bajo las cuales se asignan los recursos productivos óptimamente entre usos competitivos de forma que se maximice la utilidad de los consumidores, en un modelo con información completa. Además es una teoría de la decisión en la que primero se fija la función objetivo, a continuación se eligen las variables y, por último, se consideran las restricciones. Por el contrario, la teoría austriaca es una investigación sobre las restricciones de la actividad económica y los cambios en las mismas, todo ello en un mundo con incertidumbre $y$, aunque introduce la utilidad marginal, no es la clave de su investigación (Streissler, 1972, 426-27). 
Son varios los aspectos de las teorías de Menger que sustentan estas opiniones. Por ejemplo, el énfasis que pone en la dimensión temporal de los fenómenos y los problemas económicos; la clasificación vertical de los bienes en rangos inferior y superior, desde los bienes de capital más alejados del consumo, hasta los bienes finales, lo que sugiere un acercamiento a la división clásica entre materias primas y productos finales, y en consecuencia, un distanciamiento con los supuestos neoclásicos (Streissler y Weber, 1973, p. 229; Huerta de Soto, 2000).

La obra de Menger se centra en los aspectos «imperfectos» de la economía y el objeto de su estudio es una economía que siempre está en desequilibrio. Llega a rechazar la ley de la unicidad del precio en equilibrio (Streissler, 1972, p. 439; Jaffé, 1976, p. 153). Su idea es que un bien no tiene en un momento determinado el mismo precio en todos los lugares, menos aún en momentos cercanos en el tiempo. Incluso en mercados bien ordenados sólo se pueden determinar precios límite, dentro de los cuales el precio se determina mediante procesos de negociación. El que la demanda y la oferta no sean iguales es una característica habitual de la economía, y en consecuencia el intercambio tiene costes; este hecho es para Menger un argumento más para rechazar la idea del precio de equilibrio (Streissler 1972, 436-437).

Por otro lado, su concepto de Utilidad Marginal no es exactamente igual al de Jevons y Walras (Kirzner, 1987, p. 146). Hay que tener en cuenta que Menger no se inspiró en las mismas fuentes que estos autores, sino que con independencia de la fuerte influencia que ejercían los economistas alemanes Knies y Roscher en las universidades austriacas y que alcanzó a Menger, su maestro fue Joseph Kudler, de quien tomó los conceptos de partida para elaborar su teoría del valor (Kauder, É., 1961, p. 75). Además de Kudler, también influyeron en él Eberhard Friedländer y Karl Rau. Este último fue uno de los primeros economistas en estudiar la estabilidad del equilibrio del mercado mediante gráficos y fórmulas algebraicas (Zuidema, J.R., 1988, p. 14).

Menger no sólo nunca utilizó el término «marginal», sino que además evitó siempre el término «utilidad» y cuando lo empleó siempre advirtió lo inadecuado que le parecía su uso porque llevaba a confundir los conceptos de utilidad, grado de utilidad y 
valor de uso (Menger, C., 1983, n.5, p. 105; Howey, R.S., 1960, p. 40). La utilidad para Menger es la capacidad que tienen los bienes de satisfacer las necesidades humanas. Tanto los bienes económicos como los no económicos son útiles pero se diferencian en que los primeros son escasos y los segundos no (Menger, C., 1983, p. 107). Para Howey, aunque no lo expuso explícitamente, esta actitud se debe a que Menger no concebía la utilidad como la medida del valor de las cosas ya que es una cualidad no susceptible de graduarse, a diferencia del valor. Por eso, siempre utilizó el concepto de «importancia de la satisfacción» porque aunque la satisfacción tampoco es divisible en grados, la importancia que tiene a los ojos de los individuos sí. De esta forma, para Menger el valor de un bien es igual a la importancia (o significación) de aquella necesidad para cuya satisfacción el individuo necesita disponer del bien en cuestión. Esta significación de la satisfacción de la necesidad es de carácter subjetivo y, por tanto, está sujeta a error (Menger, C., 1983, pp. 132-35).

Precisamente, otra divergencia entre Menger y los otros pioneros es la caracterización del homo oeconomicus como un individuo lleno de incertidumbre y que se equivoca, incapaz de calibrar sus decisiones con el objeto de maximizar su satisfacción, en contraste con el individuo racional que nos presenta la teoría neoclásica (Jaffé, W., 1976, p. 154; Streissler y Weber, 1973, p. 229). Es bastante significativo el hecho de que el propio Menger no quisiera que le incluyeran dentro de la Escuela Marginalista (Howey, R.S., 1960, p. 142)

Sin embargo, la diferencia más llamativa entre Menger y Jevons y Walras es su rechazo al concepto de divisibilidad sin límites de los bienes y en consecuencia al cálculo diferencial, de forma que en la teoría económica nada es diferenciable; para Menger, no existen funciones continuas (Gramm y Walsh, 1972, p. 52). Streissler afirma que Menger había incluido en su obra prácticamente todos los conceptos que dificultaban la aplicación del cálculo marginal; su rechazo del análisis del equilibrio explica su reparo al empleo de las matemáticas (Streissler, 1972, p. 439-440). Como veremos más adelante, los sucesores de Menger heredaron esta renuencia al uso de funciones continuas. 
Jevons y Walras se dieron cuenta de que el supuesto de continuidad impedía que sus modelos se ajustaran a la realidad y, de hecho, intentaron explicar el caso de bienes no divisibles en algún apartado de sus obras, pero sólo de forma secundaria (Howey, R.S., 1960, p. 45).

III.

\section{LA METODOLOGÍA DE CARL MENGER}

En sus dos obras principales Principios de Economía Política (1871) e Investigaciones sobre el método de las Ciencias Sociales (1883) Carl Menger demuestra su preocupación por los problemas metodológicos. El primer libro constituyó un intento de aplicar el análisis teórico a la economía dentro de la tradición germánica y chocó frontalmente precisamente con esa tradición historicista que emanaba de Alemania e invadía las universidades de Austria (Streissler y Weber, 1973, p. 240). Menger, que en principio no trataba en absoluto de revolucionar la ciencia, se dio cuenta de que no sólo se rechazaba su libro sino cualquier atisbo de teoría en el campo de la economía (Schumpeter, J.A. 1971, pp. 28-29). La controversia entre la Escuela Histórica y Menger conocida como Methodenstreit no era un malentendido fruto del dogmatismo de ambos sino que mantenían posturas epistemológicas irreconciliables (Mildford, K., 1995, pp. 28-29).

Frente a la obsesión por la investigación histórica, Menger distinguía entre enunciados analíticos, que se refieren a conceptos y dan lugar a leyes exactas y enunciados sintéticos que se refieren a objetos y dan lugar a leyes empíricas. Estas leyes empíricas no se diferencian de las exactas en que el proceso de obtención sea deductivo o inductivo, puesto que ambas son resultado de procesos mixtos, sino en la naturaleza formal de las mismas y el tipo de análisis al que llevan (Zuidema, 1988, pp. 18-19; Oller, J.L., 1973, p. 24). Las leyes empíricas tienen un bajo nivel de abstracción y relacionan fenómenos observables, por lo que admiten ser contrastadas con la realidad. Las leyes exactas tienen un alto nivel de abstracción y ponen de manifiesto 
relaciones necesarias entre conceptos, no admiten contrastación empírica porque encuentran en sí mismas el fundamento de su validez (Hutchison, T.W., 1973 y 1981, p. 179). Emil Kauder, estudioso de Menger y su escuela, apunta que el significado de las llamadas «leyes exactas» de Menger no se corresponde con el concepto matemático usual de precisión absoluta que puede alcanzarse solamente mediante el uso de datos medibles y formulaciones matemáticas. Menger expresa plenamente lo que quiere decir cuando escribe a Walras y las denomina «lois fixes»; es decir, son enunciados sobre secuencias invariables que no están influidas por el tiempo ni por el lugar. Más que construcciones mentales individuales son descripciones de configuraciones eternas en la vida económica (Kauder, É., 1957, pp. 416-417).

Las ciencias naturales formulan leyes exactas y su método es análogo al de las ciencias sociales, con la diferencia de que éstas últimas toman como punto de partida los individuos y sus propósitos, mientras que las primeras están en desventaja porque parten del estudio de los átomos y las fuerzas, que no tienen un referente inmediato conocido.

Aunque defiende la utilización del método deductivo reconoce sin reservas la utilidad del método inductivo (Bloch, 1940, p. 443). Pero, a diferencia de Roscher, Menger defiende que las leyes empíricas son de tanta utilidad en Economía como las teóricas, lo que sucede es que cada tipo de ley se aplica para un tipo de investigación diferente. Es curioso que Menger se interesara por el estudio teórico de la ley del precio en 1869, cuando trabajaba haciendo informes en la revista de carácter económico editada por el Ministerio Wiener Zeitung, para lo cual recogía datos sobre las condiciones del mercado; observó que los precios de los bienes no venían determinados como los estudios tradicionales de economía le enseñaban, sino que los hombres de negocios sólo tomaban en consideración la práctica del mercado $^{1}$.

1 Según recoge Hayek en la página 20 de la «Introducción» a los Principios de Economía Política de Menger, Wieser afirmaba que Menger se lo había comentado. 
Para Menger la Economía Política se divide en tres ramas: la Teoría Económica, las Ciencias Históricas, como la Estadística o la Historia Económica, y las Ciencias Prácticas, como la Ciencia de la Hacienda o la Política Económica (Oller, 1973, p. 68). Define la Teoría Económica como una ciencia apriorística en la que se proponen modelos conceptuales que no necesariamente tienen que tener un correlato empírico inmediato, son simplemente «retratos de la realidad que subyace a la apariencia cotidiana» (Oller, 1973, p. 19). Menger consideraba que la economía teórica no debe ocuparse de las interrelaciones de los fenómenos económicos, sino que su objetivo principal debe ser descubrir las relaciones de causalidad que los ligan.

Para lograr este objetivo hay que razonar partiendo de lo más simple para terminar estudiando los fenómenos mas complejos, y además, es necesario buscar la causa de cada paso del razonamiento en el paso anterior (Antonelli, É., 1953, pp. 271272). De esta forma, las conclusiones a las que llegamos en cada eslabón de la cadena deben ser originadas en el eslabón anterior. Este argumento es crucial porque, como indica Emil Kauder, constituye el principal punto de ruptura entre el método mengeriano y el matemático; es por esta herencia aristotélica por lo que Menger rechaza la interdependencia, el equilibrio, las ecuaciones y las relaciones funcionales. El principio causal es totalmente incompatible con las matemáticas (Kauder, 1965, p. 70).

Además, para Menger, los cambios en los fenómenos contemplados dejan inalterada su esencia y solamente amplían el ámbito de la investigación. El concepto de «cambio» como realización de algo que estaba, en potencia, en la esencia del fenómeno y la definición de la Historia como descripción de estos cambios, explican que para Menger los fenómenos económicos tenían que contemplarse en su desarrollo temporal.

Partiendo de estos principios y a modo de resumen, José-Luis Oller establece una serie de postulados que definen el esquema conceptual de la teoría económica mengeriana. En primer lugar, hay un conjunto de fenómenos que son consustanciales a la actividad económica e independientes de la estructura social; este conjunto está formado por la necesidad, la escasez y la capacidad de satisfacer las necesidades por parte del individuo. 
Además existe otro tipo de fenómenos que surgen en cualquier forma de actividad económica con un mínimo de desarrollo como el cambio, el precio, el mercado, etc., y que aparecen con un mayor o menor grado de complejidad según los distintos tipos de economía. Para comprender cada uno de los fenómenos cambiantes se han de poder explicar en su desarrollo genético-causal en cada una de las etapas del proceso.

Por último, la explicación del paso de un estadio a otro debe reducirse a relaciones entre los factores originarios que hemos mencionado en primer lugar (escasez, necesidad y capacidad de las personas de satisfacer sus necesidades). Menger llama a este método analítico-causal o analítico-sintético y afirma que es el único adecuado para lograr el objetivo de la teoría económica.

Respecto a la utilidad de las matemáticas como instrumento para la investigación teórica, tal y como explica a Léon Walras en una de las últimas cartas que se intercambiaron, son una ciencia auxiliar válida únicamente para ilustrar gráficamente una demostración o como mera forma de exposición, pero no como instrumento de análisis capaz de hacer avanzar la investigación (Antonelli, 1953, pp. 273-274). Puesto que mediante el método analítico-sintético (o genético-causal) es necesario, en primer lugar, llegar al conocimiento de las esencias de los elementos más simples, que subyacen a la realidad y son independientes de la voluntad humana, para descubrir la ley que rige los fenómenos más complejos derivados de ella, las matemáticas no pueden más que jugar un papel secundario, puesto que, según Menger, se ocupan del estudio de las magnitudes (Antonelli, 1953, p. 280). Las matemáticas son epistemológicamente incapaces de descubrir las esencias (Alter, M., 1990, p. 95). Fue precisamente esta actitud de Menger hacia las matemáticas la que provocó el final de la correspondencia que hasta entonces habían mantenido los dos autores.

También se sabe que criticó el libro de Gossen llamado Entwicklung der Gesetze des menschlichen Verkehrs (1854), y que Menger leyó en 1886, por su enfoque hedonístico, su énfasis en la importancia del trabajo y la aplicación de las matemáticas al ámbito de la psicología (Hayek, 1991, n. 60, p. 370). 
En 1889 comentó la teoría de los precios de los economistas Auspitz y Lieben y, aunque se niega, en principio, a comentar el valor de la exposición matemática de los principios doctrinales de la Economía Política, apunta como algo negativo el que los autores utilizaran el método matemático no sólo como medio de exposición sino como método de investigación (Hayek en Menger 1983, n.6, p. 18).

En las anotaciones no publicadas al libro Grundsätze der Volkswirtschaftslehre (1863) de Karl Rau recogidas y estudiadas por Emil Kauder, Menger utiliza figuras geométricas y fórmulas sencillas para explicar el intercambio aislado entre dos individuos. En esta revisión que data de 1867, utiliza un ejemplo que más tarde emplearía en sus Principios de 1871, aunque algo simplificado (Menger 1983, n.2, p. 159). Lo que Menger trata de ilustrar es cómo en el caso de dos individuos aislados cada uno de los cuales tiene un exceso del bien que el otro carece, el intercambio favorece a ambos. El origen de esta teoría la encontramos en el borrador de los Principios y en las anotaciones que hizo al libro de Karl Rau en 1867 (Kauder, 1961, p. 75). Para ello representa los bienes que cada individuo A y B posee mediante triángulos, de la siguiente forma:

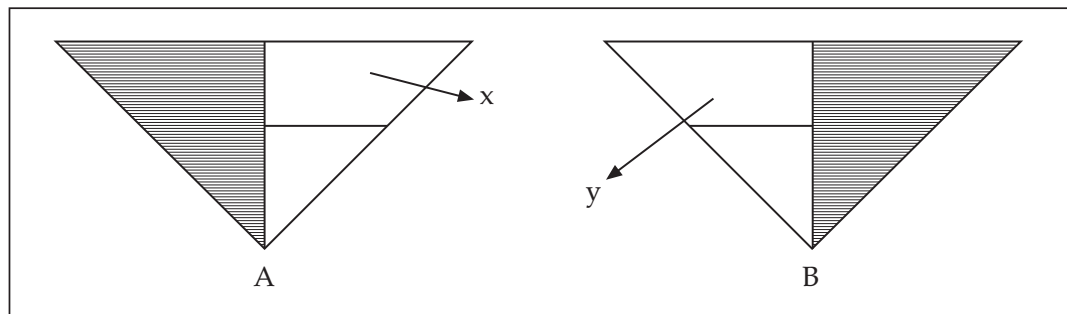

Menger no escribió en ellos notación alguna, pero de acuerdo con el ejemplo que tratan de ilustrar, Emil Kauder añadió una notación que aparece entre paréntesis (Kauder, 1961, pp. 76-78).

Menger supone dos individuos A y B, que poseen dos bienes $a$ y $b$ respectivamente, y que $x$ es la cantidad adicional del bien poseído por el individuo A, e $y$ es la cantidad adicional del bien poseído por el individuo $\mathrm{B}$. El individuo A ofrecerá la cantidad $x$ de su bien $a$ contra una cantidad indeterminada, unas unidades 
de $y$, el excedente del bien $b$ propiedad del individuo $\mathrm{B}$, y viceversa. Para $A$, vale más lo que posee $B$ que lo que posee él mismo, es decir $b+y>a+x$ y para B será al revés. Seguirán demandando y ofreciendo en la medida en que ambos ganen con el intercambio (Kauder, 1961, p. 77). En el ejemplo de los Principios, Menger denomina $W$ al valor que tiene para el individuo A una unidad adicional del bien $a$ y $\mathrm{W}+x$ al valor de una unidad adicional del bien $b$ para el mismo individuo; de la misma forma $\mathrm{w}$ es el valor que una unidad de $b$ tiene para $\mathrm{B}$ y $w+y$ al valor que tiene una unidad de a para el individuo B. Cuando intercambian $1 a$ por $1 b$, el individuo A se encuentra en una situación en la que ha ganado $x$ y B ha ganado $y$ (Menger 1983, n. 2, p. 159 de nuevo). Es decir, el mayor valor que un individuo da subjetivamente al bien que no posee es exactamente la cantidad que está dispuesto a ceder de su bien para conseguirlo.

En las páginas siguientes del libro, Menger incluyó una tabla numérica con el objeto de facilitar la comprensión de la investigación y para ilustrar la escala de la «significación escalonada de las necesidades» de los individuos que es descendente, según Menger, en proporción aritmética. Pero en una nota aclara que las cifras no expresan cantidades absolutas sino relativas, de forma que si 20 y 40 son la significación de la satisfacción de dos necesidades diferentes, eso sólo quiere decir que la segunda tiene el doble de importancia para el individuo (Menger, n.3, p. 162).

Sin embargo, si bien Menger acepta que es posible relacionar los valores subjetivos de los bienes estableciendo una jerarquía, sostiene que esta comparación es demasiado inexacta como para tomarla como medida del valor.

IV.

\section{LA FORMACIÓN MATEMÁTICA DE MENGER}

No hay un acuerdo entre los economistas que han estudiado a Menger en lo que se refiere a su formación matemática. Si bien era jurista, en los Gymnasium de la antigua Austria se impartían clases de matemáticas. No se sabe el nivel de dificultad de la enseñanza, pero es uno de los argumentos que aducen algunos 
autores para afirmar que tenía formación matemática (Kauder, 1957, p. 412 y 1965, p. 63). Según la información aportada por su propio hijo, se ha encontrado un cuaderno de notas fechado en 1867-68 en el que hay un par de páginas en las que incluye gráficos en los que la utilidad viene dada por debajo de una gráfica lineal, que representa la utilidad marginal. En cualquier caso, Menger (hijo) opina que su padre nunca tuvo un conocimiento operativo del cálculo. También cuenta que aprendió cálculo diferencial de forma autodidacta en la década de 1890 (Menger, K. 1985, pp. 55-72). Menger mostró su interés por las matemáticas y en especial por las obras de Cournot en una carta escrita en 1911 a Ismar Feilbogen. Incluso le aconsejó a Walras, en la misma carta en la que le explicaba su rechazo a las matemáticas, la lectura de autores que habían aplicado ese método con anterioridad a Cournot, Jevons y Gossen, y cita incluso las páginas de las obras en las que emplean las matemáticas; entre esos trabajos nombra los Principes d'Economie Politique (1801) de Canard, el Saggi Economici (1827) de Fuoco y Der isolierte Staat (1842-50) de von Thünen (Antonelli, 1953, pp. 272-273). Otros autores como Streissler consideran que sabía las suficientes matemáticas como para que su rechazo a su empleo se debiera sólo a motivos metodológicos y no a su ignorancia, pero la base de su argumento es que provenía de una familia de «mentes matemáticas» puesto que su hijo y su hermano eran matemáticos (Hayek en Menger, 1983, p. 18); simplemente los instrumentos matemáticos que tenía a su alcance, el cálculo, sólo era adecuado para la descripción de las situaciones de equilibrio, que no le interesaban.

$\mathrm{V}$.

ALGUNAS POSIBLES APORTACIONES DE LA MATEMÁTICA ACTUAL

Hoy en día sabemos que es posible utilizar una parte de las matemáticas en el análisis de la escala de la 'significación escalonada de las necesidades' de la que hablaba Menger. J. Huston McCulloch estudió en 1977 la teoría ordinal de la utilidad 
marginal austriaca utilizando las matemáticas de conjuntos ordenados. Dada una ordenación (según su significación) de las necesidades satisfechas por una cantidad determinada de un bien en conjuntos, considera que, si $\mathrm{P}_{\mathrm{n}}$ es el conjunto de necesidades que es satisfecho por $\mathrm{n}$ unidades de un bien y $\mathrm{P}_{\mathrm{n}-1}$ es el conjunto de necesidades satisfecho por $\mathrm{n}-1$ unidades de dicho bien, el conjunto diferencia $P_{n}-P_{n-1}$ será el conjunto de necesidades que dependen de la unidad de bien n-ésima; a ese conjunto diferencia lo llama uso marginal. A partir de ahí, McCulloch define la Ley Austriaca del Uso Marginal según la cual, la utilidad del incremento de un bien está determinada por la posición de su uso marginal en la escala de los conjuntos de necesidades. La consecuencia inmediata de esta Ley es el hecho de que si tenemos una cantidad mayor de un bien, las necesidades que dependen de él tendrán un menor rango en la escala y por lo tanto su utilidad marginal será menor; según esta formulación el principio de disminución marginal de la utilidad es un teorema no un supuesto como en las teorías de Walras y Jevons. Como los austriacos no creían en la indivisibilidad infinita de los bienes, la utilidad se refiere a incrementos no infinitesimales. Además, la utilidad marginal no es la diferencia aritmética de los niveles de utilidad sino el conjunto diferencia de las necesidades. Para McCulloch, los austriacos operaban con conjuntos implícitamente y tal vez ni ellos mismos lo sabían; esto es posible porque el álgebra de conjuntos no es más que la formalización de categorías elementales de la lógica y la notación de conjuntos es relativamente reciente (McCulloch, 1977, pp. 249-256). Sobre esta base continúa analizando las curvas de indiferencia, los bienes complementarios y la elasticidad de sustitución, así como el índice de utilidad del modelo von Neumann-Morgenstern y los problemas que se plantean cuando conservamos el carácter ordinal de la utilidad (McCulloch, 1977, pp. 257-277).

Entre 1873 y 1892, Menger corrigió los Principios de Economía Política sobre una copia de la edición de 1871 que se conserva en su biblioteca privada. En ella hay anotaciones sobre la teoría del capital, de la productividad y de la renta nacional en las que incluye fórmulas simples. Por ejemplo: si durante un período de tiempo un granjero necesita $x$ cantidad de semillas y granos e $y$ 
de trabajo, y si $y=2 x$, entonces después de algunos meses cosechará una cantidad $6 x$. Como sus gastos son de $3 x=x+y$, siendo $y=2 x$, trás la cosecha tendrá un beneficio de $3 x$, es decir, $6 x$ de producto bruto menos $3 x$ de coste. Si el granjero no tiene una cantidad adicional $x+y$ para esperar a la cosecha, debe renunciar a una ganancia de $3 x$. En la introducción de la segunda edición del libro que estaba preparando utilizó un cálculo similar para determinar el capital presente y futuro (Menger, 1961, p. 79).

Emil Kauder, quien ha consultado la biblioteca de Menger actualmente propiedad de la universidad de Tokio, estudiando detenidamente todos los escritos publicados e inéditos, se sorprende ante esta actitud de Menger, sobre todo dadas las repetidas críticas que había hecho sobre el empleo de las matemáticas en economía. Por otro lado, llama la atención que no se diera cuenta de las posibilidades de esta ciencia auxiliar una vez que empezó a recurrir a ella. Otro especialista, Karl Borch hace hincapié en el tratamiento simétrico que hizo Menger de tiempo e incertidumbre careciendo de conocimientos matemáticos, porque es una relación que no resulta tan evidente para los economistas no matemáticos de la época y que fue tratada sólo por los actuarios (Borch, 1973, pp. 61 y ss.).

En los años en que Menger escribió había actuarios que trabajaban en compañías de seguros y trataban estos problemas de forma matemática pero no hay signos de contacto alguno entre ellos y los austriacos. Lo que Menger estudia es la incertidumbre acerca del output que se puede obtener a partir de un input determinado. Matemáticamente, si la función de producción normal se representa como $\mathrm{y}=\mathrm{f}\left(\mathrm{x}_{1}, \mathrm{x}_{2}, \ldots \mathrm{x}_{\mathrm{n}}\right)$, Menger habría expresado su función mediante una relación estocástica como por ejemplo: $\operatorname{Pr}($ output $=\mathrm{y})=\mathrm{f}\left(\mathrm{y} / \mathrm{x}_{1} \ldots \mathrm{x}_{\mathrm{n}}\right)$ que es la función de distribución de la probabilidad condicionada. Este paso lo dió el austriaco Gerhard Tintner en 1941 (Borch, 1973, p. 66). A principios del siglo XX existía en Viena una importante escuela de matemáticos actuarios encabezados por Emanuel Czuber y Blaschke, que se dedicaban al estudio de los mismos problemas que BöhmBawerk y la Escuela Austriaca, sin hacer referencia a la teoría económica. La única mención a Menger la hizo Czuber en 1902, cuando afirmó que el fundamento de la nueva teoría del valor 
antes creada por Menger, Jevons y Walras era el Principio de Bernoulli. El matemático Daniel Bernoulli estudió en 1732 el riesgo que supone para un individuo apostar sucesivas sumas de dinero en un juego de azar según la expectativa de ganancia. Bernoulli estableció que la medida del riesgo (o de la utilidad del beneficio) de cada apuesta venía dada por la «expectativa moral» que tenía en cuenta la fortuna previa del jugador como factor que disminuía la utilidad esperada de la ganancia, en lugar de la esperanza matemática (Borch, 1973, p. 73). De acuerdo con Streissler, las matemáticas actuales sí pueden resolver los problemas que preocupaban a Menger, por ejemplo, la teoría de los procesos estocásticos, etc (Streissler, 1972, p. 440).

De hecho, recientemente, se ha desarrollado una rama de las matemáticas que se dedica a investigar acerca de la teoría de la decisión de manera más elaborada que en la época de Menger. Se trata de la Teoría de la Decisión Multicriterio que intenta solucionar los problemas que afloran en las tomas de decisión cuando existen objetivos múltiples y, a menudo, conflictivos entre sí. El objetivo es obtener una escala natural para cuantificar las preferencias expresadas verbalmente a partir de la observación de la pauta habitual del juicio comparativo humano en áreas independientes como la historia, la planificación y la psicofísica. Esta teoría surge del rechazo hacia la investigación operativa basada en la optimización. La optimización matemática parte del postulado según el cual, en cualquier situación que entraña una elección, existe al menos una decisión que, disponiendo del tiempo y los medios necesarios, puede se objetivamente demostrada como óptima, y esto permaneciendo neutral en relación al proceso de decisión en sí. Para los teóricos de la Decisión Multicriterio, es imposible tomar decisiones con esos supuestos, además, la optimización supone que la decisión resultante es indiscutible e irreversible y que el decisor es plenamente responsable y tiene información perfecta. Otro argumento en contra de la optimización es que las técnicas de cálculo se han desarrollado por encima de lo razonable en detrimento de una formulación correcta del problema, de una evaluación crítica de los datos y de una revisión permanente del modelo. Finalmente, consideran que las acciones potenciales, a menudo son juzgadas 
por sus consecuencias que, en la práctica, son heterogéneas y mal definidas. De manera que, en ocasiones, la falta de instrumentos de cuantificación adecuados lleva a rechazar determinadas consecuencias que se dan en la realidad. En cierto sentido, estas críticas se aproximan al punto de vista austriaco expuesto más arriba.

Hay dos principales corrientes según el enfoque dado a la Decisión Multicriterio. Por un lado está la escuela americana encabezada por Saatsy y el proceso de análisis de la jerarquía que trata de incorporar el juicio humano subjetivo de diferentes maneras. Por otro lado está la corriente francesa liderada por Bernard Roy y el sistema binario de superioridad de rango, ELECTRE. En ambos casos se trata de la evaluación de un número finito de alternativas $A_{1}, A_{2}, \ldots, A_{n}$, según un número finito de criterios $\mathrm{C}_{1}, \mathrm{C}_{2}, \ldots, \mathrm{C}_{\mathrm{m}}$, por un único decisor o «cuerpo decisorio", teniendo en cuenta que la elección de una alternativa tendrá consecuencias conocidas de antemano ya que se supone que la incertidumbre respecto al futuro estado del mundo es insignificante.

La corriente americana contempla cuatro temas principales dentro de esta línea de investigación: la programación matemática con múltiples criterios, las alternativas discretas multicriterio, la teoría de la utilidad multiatributo y la teoría de la negociación. De todos ellos, por su posible relación con la teoría económica mengeriana, nos interesan los dos últimos.

La Teoría de la Utilidad Multiatributo parte del trabajo de von Neuman y Morgenstern de 1944. Esta teoría de suele definir como la valoración y adecuación entre las funciones de utilidad y la teoría de la probabilidad. Las funciones de utilidad se emplean para clasificar decisiones alternativas y facilitar la elección, o bien como funciones objetivos en los problemas de programación matemática. Implícitamente, la teoría de la utilidad es una teoría multiatributo. En ella se emplea un muestreo aleatorio para evaluar las funciones de utilidad equivalentes a los resultados fijados. Por regla general, este muestreo incluye al menos dos resultados, el mejor y el peor de todos los posibles.

La Teoría de la Negociación es interpretada desde puntos de vista muy diferentes. Se considera bien como una rama de la 
teoría de juegos, bien en relación con la teoría de la utilidad, como parte de la psicología, del análisis del comportamiento organizativo e incluso como parte de la ciencia política (Zionts, en Goicoechea, Duckstein y Zionts, eds., 1992, pp. 34-35.). Tiene claras limitaciones importantes pero puede resultar útil para elaborar sistemas de apoyo a la toma de decisiones, encaminados a ayudar a los negociadores a comprender y estructurar sus propias posiciones así como también a determinar una función de utilidad que se pueda emplear en la evaluación de decisiones alternativas.

La escuela francesa, por su parte, defiende el complejo proyecto ELECTRE. Este sistema se basa en la consideración de un par de alternativas a las que o bien se les asigna un valor numérico si es cuantificable - por ejemplo, el coste monetario- o, en caso contrario, se representa su valor en una escala cualitativa. Es necesario introducir unos umbrales en la escala de valor para obtener información necesaria respecto a las preferencias y así poder determinar si una opción es indiferente a la otra, si existe una preferencia débil o una preferencia estricta entre una y otra. De esta manera el decisor establecerá el rango de la relación entre dos opciones. El paso siguiente consiste en la asignación de un factor de importancia a los criterios de su elección y en la elaboración de una análisis de sensibilidad del modelo. Finalmente, para cada par de alternativas, el sistema ELECTRE asigna un índice de concordancia y otro de discordancia, que reflejan respectivamente las evidencias a favor y en contra de la superioridad de rango de una alternativa frente a la otra.

Estos tipos de análisis, sin embargo, presentan algunos problemas. Por un lado, el sistema ELECTRE no siempre puede identificar la alternativa preferida y se limita a ofrecer un núcleo de alternativas más importantes. Por otro lado, un análisis de sensibilidad del modelo para determinar la susceptibilidad de cambio de los resultados cuando se alteran uno o varios de los factores y condiciones iniciales, basado en una diversidad de escalas geométricas muestra que, tanto la corriente francesa como la americana, no son capaces de ordenar sin ambigüedad las alternativas en una clasificación subjetiva de las preferencias. (Lootsma, F.A., en Goicoechea, Duckstein y Zionts, eds., 1992, p. 253 y ss.) 
A pesar de sus limitaciones, la teoría de la decisión multicriterio se esá empleando en la planificación de la mejora de la calidad de vida, el desarrollo de programas de ordenador y estudios acerca del suministro de agua (Goicoechea, Stakhiv y Li, en Goicoechea, Duckstein y Zionts, 1992, pp. 2-16).

Todas estas aportaciones no terminan de contentar a los economistas de la escuela austriaca actual quienes plantean como algo inalcanzable el reto de conciliar el empleo de las matemáticas modernas en el análisis económico de los procesos de acción (que no de decisión) humana.

VI.

\section{LOS PRIMEROS DISCÍPULOS DE MENGER}

Los principales seguidores de Menger fueron sus discípulos (aunque no alumnos) Eugen von Böhm-Bawerk y Friedrich Frieherr von Wieser, quienes no sólo difundieron las teorías del maestro, sino que también las completaron en algunas partes esenciales (Kirzner, I., 1987, p. 145-46). A pesar de las diferencias de matiz en algunas de sus teorías respecto a las de Menger, conservaron el método y, en concreto, la postura reticente hacia las matemáticas.

Böhm-Bawerk (1851-1914) no trató los problemas metodológicos tan extensamente como Menger (Seligman 1963, p. 296 y 1981, p. 204). Sí definió cúal era su punto de vista que a grandes rasgos coincide con el del maestro, sobre todo en una carta de Walras, con el que discutía la teoría del interés, en la que el francés afirma tajantemente que el rechazo manifiesto que BöhmBawerk le había expresado hacia la utilización del método matemático por parte de Walras en economía hacía su acercamiento teórico imposible (Jaffé, ed., 1965, pp. 316-317).

En sus cartas con Walras se observan otros puntos de divergencia importantes entre el enfoque austriaco y el enfoque matemático. En primer lugar, su desacuerdo en lo referente a la continuidad de la función de utilidad (Hutchison, 1967, pp. 175-176). Böhm-Bawerk le expone a Walras que de acuerdo con la teoría Austriaca del valor las variaciones en la utilidad son discretas; 
como réplica, Walras remite a Böhm-Bawerk a la «ley de los grandes números» enunciada por Cournot, según la cual variaciones de la oferta y la demanda discontinuas a nivel individual se traducen en cambios continuos de la oferta y la demanda generales. En su respuesta, Böhm-Bawerk no nombra dicha ley, por lo que no sabemos si realmente la conocía. Sin embargo, y a diferencia de Menger, Böhm-Bawerk admite que existen algunos bienes infinitamente divisibles lo que implica que su función de utilidad es continua, pero él prefiere tratarlos como no infinitamente indivisibles aunque no explica por qué. Es más, afirma que si hubiera conocido exactamente las teorías de Walras a este respecto con anterioridad, habría estudiado la ley de los precios para ambos casos, divisibilidad e indivisibilidad; sin embargo, tampoco lo hizo más adelante (Jaffé, ed., 1965, pp. 193-195).

En su libro Positive Theorie des Kapitales (1889) expresa su opinión sobre la teoría del capital walrasiana diciendo que contiene notables pasajes pero lamenta el que esté expresada en lenguaje matemático porque le parece problemático y difícil, y añade que «la concepción de la Economía Política como una ciencia eminentemente matemática» es una idea con la que nunca estará de acuerdo (Jaffé, ed., 1965, p. 317).

También expresa su recelo respecto a la consecución del equilibrio. Para Walras, dado que el principio de proporcionalidad entre «raretés» y precios es la condición de satisfacción máxima de las necesidades de los individuos, el que el equilibrio se mantenga depende de hasta qué punto cada individuo sabe obtener y mantener su satisfacción máxima. Pero Böhm-Bawerk opina que hay excesivos «rozamientos» en este intento de buscar el equilibrio económico (Jaffé, ed., 1965, p. 183). De acuerdo con Max Alter, la defensa del método correcto por parte de Böhm-Bawerk nunca fue mucho más que «una llamada al sentido común» y su rechazo del análisis matemático se apoyaba en motivos didácticos pero no aportó argumentos metodológicos para sostenerlo (Alter, 1990, p. 226). Por otro lado, en su revisión de 1894 del libro de Wicksell Über Wert, Kapital und Rente, Böhm-Bawerk dudaba que muchos lectores siguieran las explicaciones algebraicas y geométricas en un libro que trataba de economía teórica. 
Sin embargo a lo largo de los años, Böhm-Bawerk demostró una mayor flexibilidad respecto a los economistas matemáticos. Este cambio de actitud se observa en el estudio y crítica que hizo de las diferentes teorías del capital y el interés en su libro Capital e Interés, publicado en 1884 y en cuya segunda edición de 1900 añadió un análisis de las teorías surgidas en esos años (BöhmBawerk, 1947, p. 560 y ss.). En primer lugar, llama la atención la ausencia de algún comentario sobre el método matemático de autores como Jevons o Marshall; y en segundo lugar, todavía sorprende más que acusara a Marshall de realizar un doble cálculo del interés y que le reprochara llegar a dos fórmulas diferentes que partían de premisas incompatibles entre sí para calcular el mismo concepto, presentándolas simplemente en una nota a pie de página, en lugar de «ilustrar el estado de sus opiniones mediante una fórmula», lo que según Böhm-Bawerk responde a un intento por parte de Marshall de esquivar un conflicto matemático manifiesto (Bóhm-Bawerk, 1947, n. 67, pp. 561.563). A pesar de ello, dado que él mismo no se considera lo suficientemente matemático no entra a analizar en detalle hasta qué punto Marshall ha ocultado sus incongruencias mediante la formulación matemática del tema. De estas acusaciones se deduce, por un lado, un cierto conocimiento de la economía matemática y, por otro lado, una mayor apertura que Menger a la hora de discutir temas de teoría económica desde el enfoque matemático. Como acertadamente señaló Schumpeter, asombra la seguridad y corrección con que Böhm-Bawerk utilizó formas de pensamiento esencialmente matemáticas sin recurrir ni a símbolos ni a tecnicismos matemáticos (Schumpeter, 1971, pp. 267-268). El economista holandés Heijman opina que precisamente porque no empleó las matemáticas, resulta difícil interpretar algunas partes de su obra y que a esto se debe que su teoría del interés no fuera más aceptada por los economistas de su época (Heijman, 1988, p. 89).

Friedrich Frieherr von Wieser fue el discípulo que más extendió las teorías de Menger, entre otras cosas porque sobrevivió tanto al maestro como a su amigo Böhm-Bawerk. Aunque no se interesó por la metodología en sí, Wieser expuso claramente su opinión acerca del ámbito de la utilización de las matemáticas en la teoría económica. Acepta las matemáticas en el análisis de 
los fundamentos estáticos de la teoría económica pura (Alter, 1990, pp. 221-223). En la introducción de su libro Social Economics (1914), no considera que sean de alguna ayuda para los estudiantes de economía. Las matemáticas son aplicables a la teoría del valor y la teoría de los precios en cuanto que éstas se presenten bajo el supuesto de una economía estática, porque en este caso se pueden establecer las hipótesis más abstractas e ideales; en este ámbito es el instrumento más exacto para formular los resultados. En cuanto a la economía desde el punto de vista dinámico, ni siquiera estas dos teorías se pueden presentar en forma matemática estricta. Dado que nuestro método de investigación consiste en acercarnos a los problemas de la economía pasando de una mayor a una menor abstracción, al avanzar en ella nos veremos forzados a abandonar la formulación matemática. Además, Wieser pone el ejemplo de la escuela clásica de economía que no necesitó de las matemáticas para conservar su dominio durante mucho tiempo. En las mismas teorías del valor y de los precios, las verdades fundamentales pueden expresarse sin la ayuda de símbolos matemáticos (Wieser, 1967, pp. 3-13).

En el primer capítulo, Wieser explica lo peligroso de centrarse exclusivamente en el análisis estático de los problemas, que es lo que hacían los economistas matemáticos. Según él, este análisis es útil para resaltar la visión de los problemas de la Economía Política como una unidad, pero «amenaza con introducir métodos de la Física matemática que no son los adecuados para el objeto de estudio». Admite que a pesar de los contrastes manifiestos, el proceso económico funciona con tal homogeneidad que puede idealizarse y representarse como un todo, como una unidad. Pero el análisis estático termina por simplificar tanto los supuestos relacionados con el hombre en sus actividades como los relacionados con los bienes y las necesidades, y de esta forma permite que se deduzca una condición de equilibrio perfecto; esto está en contradicción con la experiencia, ningún ajuste ha tenido como efecto tender a un equilibrio estricto.

Además el enfoque estático pasa por alto tanto la consideración de la diversidad de la riqueza natural en términos de mercancías como las diferencias en las escalas de las necesidades según los bienes y las localidades. Las simplificaciones que llevan 
consigo el análisis estático dificultan la solución de otros problemas de considerable importancia y que también requieren una explicación teórica.

También hace manifiesto su desacuerdo con el método de Walras en su libro publicado en 1893 Natural Value. En su introducción, si bien afirma que las leyes que rigen el valor son a la economía política lo que la ley de la gravedad es a la mecánica, lo que resulta tan cercano a las opiniones de Walras, pocos párrafos después sostiene que el trabajo de Walras, aunque admirable, sufre de la preponderancia del elemento matemático. Aún cuando reconoce que las leyes que gobiernan las cantidades del valor admiten expresión matemática, y que a medida que estas leyes sean más complicadas la expresión matemática es la más exacta posible, aún cuando reconoce la importante función que en este sentido pueden llevar a cabo las matemáticas, la teoría del valor no se limita a ser una ley de la cantidad sino que debe aclarar el concepto del valor, describir las formas en las que se manifiesta, mostrar su conexión con muchos otros fenómenos económicos, y para todo ello se necesita «una filosofía del valor que no necesita números sino palabras» (Wieser, 1971, p. xxxiii). Después de estas explicaciones se entiende por qué Wieser consideraba las matemáticas como una ciencia auxiliar de la teoría económica.

Menger abandonó la Universidad en 1903 y le sucedió Wieser. Hasta su muerte en 1914, el principal representante de la Escuela Austriaca fue Böhm-Bawerk a través de su seminario, que llegó a ser famoso como centro intelectual de la Escuela Austriaca. Entre sus alumnos estaba Joseph Alois Schumpeter y Ludwig von Mises, ex-alumno de Böhm-Bawerk en la Universidad (Hutchison, 1981, p. 200). Von Mises creó más adelante su propio seminario privado, que se reunía en la Cámara de Comercio y al que acudían jóvenes economistas, filósofos y sociólogos. Entre los economistas destacaban Friedrich von Hayek, Oskar Morgenstern, G. Haberler y F. Machlup. Wieser dejó en 1926 a Hans Mayer como sucesor en la universidad. Éste formó un grupo de seguidores entre los que destaca Erich Streissler, quien en 1968 se hizo cargo de la cátedra y que hoy en día sigue estudiando cultivando la historia del pensamiento económico austriaco (no así su actual programa de investigación). Mayer también rechazó explícitamente 
el empleo de las matemáticas con los mismos argumentos que Wieser (Hutchison, 1981, p. 207; Mayer, 1994). Para Streissler el rechazo de sus maestros Wieser y Mayer a la economía matemática se debe a que los fenómenos económicos son en esencia discontinuos y discretos y el cálculo diferencial de la época no podía tratarlos de forma adecuada (Streissler, 1973).

VII.

\section{CONCLUSIONES}

La metodología de Carl Menger no era completamente contraria a la economía matemática, sino más bien al modo en el que se aplicaban entonces al análisis económico y al tipo de las matemáticas que se empleaban. Tampoco Wieser o Böhm Bawerk mostraron una actitud tan tajante contra las matemáticas como en un principio podría considerarse si bien ambos autores también criticaron el modo en el que se aplicaban y la rigidez de esa parte de las matemáticas.

La discrepancia sigue viva, en parte porque la teoría económica mantiene modelos e instrumentos 'rancios'. Seguramente la solución no consiste en descalificar por completo la economía matemática sino en dejar que la teoría económica sea más permeable a los avances de las ciencias que complementan la nuestra, como las matemáticas y la psicología, y desde luego, tener en cuenta las restricciones que implican cuidadosamente en lugar de hacer uso sistemático de ellas en cualquier ocasión.

Por otro lado, hay que plantearse que como toda ciencia, la economía se basa en la explicación de la realidad mediante ideas simples a partir de la abstracción. Esta idea no es ajena a Menger. La pega del fundador es el restringir la teoría económica al estudio del proceso genético-causal, no la existencias de leyes inmutables y simples que expliquen la realidad.

Sin embargo, los sucesores de Menger plantean más problemas (Huerta de Soto, 2000). El lenguaje matemático moderno incluyendo la teoría de juegos, la topología y la teoría del caos responden a las exigencias de la lógica formal y de los fenómenos del mundo de la naturaleza. En la realidad no existen superficies perfectas sin 
rozamiento, exactamente igual que tampoco existe una función de utilidad cardinal. Ello no impide que los físicos estudien el universo a partir de supuestos más o menos restrictivos y deduzcan leyes universalmente válidas. Para los economistas austriacos, la diferencia entre el estudio de la física y el de los procesos económicos es que en éstos últimos el hombre crea y, además, tiene la capacidad de crear una estructura de tendencia al fijarse determinados objetivos. En los procesos naturales, los agentes que intervienen, sean átomos o bolas que caen por un plano, pueden moverse o reaccionar ante estímulos pero no crear o decidir en función de unos fines subjetivos.

Estos son los motivos principales por los que desde el punto de vista de los actuales economistas austriacos la economía matemática sigue siendo inválida. Aspectos como la percepción subjetiva del tiempo o la creación empresarial todavía quedan fuera del análisis ofrecido por la economía matemática, y son, precisamente, la base del proceso de mercado, es decir, de la parte esencial de la economía austriaca. La pregunta relevante es si aceptamos que la ciencia económica debe ceñirse al estudio del proceso de mercado o si abarca tanto los problemas que plantea la escuela austriaca como los que analiza la economía matemática (un mundo determinista en el que la creatividad empresarial brilla por su ausencia).

\section{BIBLIOGRAFÍA}

Alter, Max (1990) Carl Menger and the origins of Austrian Economics, Westview Press.

Antonelli, Etienne (1953), «Léon Walras et Carl Menger à travers leur correspondence», Economie Appliquée, vol. 6, Abril-Septiembre.

BLoch, Henri-Simon (1940), "Carl Menger, the founder of the Austrian School», Journal of Political Economy, vol.48, Junio. Reimpreso en Carl Menger (1840-1921), Pioneers in Economics 26, Marc Blaug ed., 1990.

BÖHM-BAWERK, Eugen von (1947), Capital e Interés [1884], edición parcial de la $6^{\mathrm{a}}$ edición alemana, México: F.C.E. 
BORCH, KARL (1973), «The place of uncertainty in the theories of the Austrian School», en Carl Menger and the Austrian School of Economics, Hicks y Weber, eds., Oxford Clarendon Press.

Bousquet, Georges-Henri (1924), «Les nouvelles tendances de l'École Autrichienne», Revue de l'Économie Politique, Septiembre.

Goicoechea, A., Duckstein, L., Zionts, S., eds., (1992), Multiple Critera Decision Making. Proceedings of the Ninth International Conference: Theory ans Applications in Business, Industry and Government, New York: Springer-Verlag.

Gram, Harvey Nelson y Walsh, Vivian Charles (1978), «Menger and Jevons in the setting of Post-von Neumann-Sraffa economics», Atlantic Economic Journal, Vol. 6, N. ${ }^{\circ}$ 4, Diciembre. HAYEK, Friedrich (1991), The Trend of Economic Thinking, Routledge. Heijman, W. (1988), «Böhm-Bawerk on time preference: economic action based on future needs», Journal of Economic Issues, Vol.15, N. ${ }^{\circ} 3 / 4$.

Howey, Richard S. (1960), The Rise of the Marginal Utility School, University of Kansas City Press.

Huerta de Soto, J. (2000), La Escuela Austriaca: mercado y creatividad empresarial, Madrid: Síntesis.

Hutchison, T.W. (1967), Historia del Pensamiento Económico, Madrid: Editorial Gredos.

Hutchison, T.W (1973), «Some themes from Investigation into method», en Carl Menger and the Austrian School of Economics, J.R. Hicks y W.Weber, eds., Oxford Clarendon Press.

Hutchison, T.W. (1981), The Politics and Philosophy of Economics. Marxians, Keynesians and Austrians, Blackwell.

Hutchison, T.W. (1981), «Carl Menger on philosophy and method», cap. 6 de The politics and philosophy of Economics. Marxians, keynesians and austrians, Oxford: Blackwell.

JAFFÉ, William, ed., (1965), Correspondence of Léon Walras and related papers, Amsterdam: North-Holland.

JAFFÉ, William (1976) «Menger, Jevons and Walras De-homogenized», Economic Inquiry, Vol. 14, N. ${ }^{\circ}$ 4, reimpreso en William Stanley Jevons. Critical Assessments, vol.III, J.C. Wood, ed. 
KAUDER, Emil ( 1957), «Intellectual and Political Roots of the Older Austrian School», Zeitschrift für Nationaloekonomie, Vol. 17. KAUDER, Emil (1961), "Freedom and economic theory», Hitsotsubashi Journal of Economics, Vol. 2, N. ${ }^{\circ}$, Septiembre KAUDER, Emil (1965), «Menger, Walras et Jevons, différences de méthode», cap. 6 de L'Utilité Marginale, Ligugé: Maison Mame. KIRZNER, Israel M. (1987), "Austrian School of Economics» The New Palgrave. A Dictionary of Economics, vol. I, Eatwell, Milgate y Newman, eds., MacMillan.

MAYer, Hans (1994), The Cognitive Value of Functional Theories of Price [1932], en Kirzner, I. (ed.), Classics in Austrian Economics, Vol. 2. Traducción española en Procesos de Mercado, Vol. 1, N. 2, Otoño 2004, pp. 143-265.

McCulloch, J. Huston (1977), «The Austrian theory of the marginal use and of ordinal marginal utility», Zeitschrift für Nationalökonomie, Vol. 37, N. ${ }^{\circ}$ 3-4.

Menger, Carl (1963), Problems of Economics and Sociology [1883], F.J.Nock, ed., University of Illinois Press. Traducción española, Método de las Ciencias Sociales, Madrid: Unión Editorial (2006).

Menger, Carl (1983), Principios de Economía Política, [1871], Unión Editorial.

Menger, Karl (1985) «Marginalismo austríaco y economía matemática», Cuadernos Económicos de ICE, N. ${ }^{\circ}$ 29-1.

Milford, Karl (1995), «Roscher's epistemological and methodological position. Its importance for the Methodenstreit», Journal of Economic Studies, Vol. 22, N. ${ }^{\text {s } 3 / 4 / 5 . ~}$

Oller, José Luis (1973), «Teoría de la ciencia», capítulo 1 de su Tesis Doctoral inédita: La teoría económica de Carl Menger. Una reinterpretación, Facultad de Ciencias Económicas y Empresariales de la Universidad de Barcelona, Febrero.

Schärlig, Alain (1990), Décider sur plusieurs critères. Panorama de l'aide à la décision multicritère. Lausanne: Presses polytechniques et universitaires romandes.

SCHUMPETER, Joseph Alois (1971), Diez grandes economistas, [1951], Madrid: Alianza Editorial.

Seligman, Ben B. (1963), Main currents in modern economics, The Free Press of Glencoe. [Traducción castellana Oikos-Tau]. 
STREISSLER, Erich (1972), «To what extent was the Austrian School marginalist?», History of Political Economy, Vol. 4, N. ${ }^{\circ} 2$.

Streissler, Erich y Weber, W. (1973), «The Menger tradition», en Carl Menger and the Austrian School of Economics, J.R. Hicks y W. Weber, eds., Oxford University Press.

WiESER, Friedrich Frieherr von (1971), Natural Value [1884], Augustus M. Kelley.

WIESER, Friedrich Frieherr von (1967), Social Economics [1914], Georges Allen\&Unwin.

ZuidemA, J.R. (1988), "Carl Menger, author of a research programme", Austrian Economics. Roots and ramifications reconsidered, parte I, J.J Krabbe, A. Nentjes y H. Visser, eds., número especial del Journal of Economic Studies, vol. 15, N. ${ }^{\circ} 3 / 4$. 